\title{
Multi-temperature Hydrodynamic Limit from Kinetic Theory in a Mixture of Rarefied Gases
}

\author{
Marzia Bisi • Giorgio Martalò • Giampiero Spiga
}

Received: 28 November 2011 / Accepted: 20 March 2012

(C) Springer Science+Business Media B.V. 2012

\begin{abstract}
Starting from the Boltzmann kinetic equations for a mixture of gas molecules whose internal structure is described by a discrete set of internal energy levels, hydrodynamic equations at Euler level are deduced by a consistent hydrodynamic limit in the presence of a two-scale collision process. The fast process driving evolution is constituted by mechanical encounters between particles of the same species, whereas inter-species scattering proceeds at the macroscopic scale. The resulting multi-temperature and multi-velocity fluid-dynamic equations are briefly commented on, and some results in closed analytical form are given for special simplified situations like Maxwellian collision kernels, or monoatomic hard sphere gases.
\end{abstract}

Keywords Kinetic theory $\cdot$ Gas mixtures $\cdot$ Multi-temperature models

Mathematics Subject Classification $82 \mathrm{C} 40 \cdot 76 \mathrm{P} 05 \cdot 80 \mathrm{~A} 17$

\section{Introduction}

It has been very well known for quite some time that the approach to equilibrium predicted by kinetic theory for gas mixtures might occur on different scales. Typically, when masses are disparate, a first Maxwellization step of the different distribution functions is followed by a slower equilibration of species (equalization of velocities and temperatures) [8]. Methods of kinetic theory, mainly a Chapman-Enskog expansion or Grad thirteen-moment approach,

M. Bisi (凶) $\cdot$ G. Spiga

Dip. di Matematica, Università di Parma, Viale delle Scienze 53/A, 43124, Parma, Italy

e-mail:marzia.bisi@unipr.it

G. Spiga

e-mail: giampiero.spiga@unipr.it

G. Martalò

Dip. di Matematica, Università di Milano, Via C. Saldini 50, 20133, Milano, Italy

e-mail: giorgio.martalo@unimi.it 
were used in this kind of analysis [7, 13, 22]. On the other hand, a multi-temperature approach naturally arises also in plasmas at high temperature [3], in the flow field around hypersonic vehicles at high altitude [15], as well as in several problems of aerothermodynamics $[16,21]$. In fact, a one-temperature gas flow description is not reliable in thermal non-equilibrium conditions when vibrational relaxations and possible chemical reactions proceed at the gas dynamic time scale, as widely investigated, especially by the Russian school [12]. In more recent years, multi-temperature fluid-dynamic models have gained significance and interest in the frame of rational thermodynamics [14]. The macroscopic theory of homogeneous mixtures has been developed from basic principles on the assumption that each constituent obeys the same balance laws as a single fluid, yielding qualitative expressions for the coefficients in the exchange rates for species momentum and energy, and emphasizing the limiting role of a single temperature approach [19]. Several papers were published along this line, dealing with average temperature, Maxwellian iteration, heat conduction $[9,18,20]$, and leading also to a quantitative application to the problem of flame structure [4]. The interested reader is referred to the review article [17] for more exhaustive information on a matter that seems to attract a broad and intensive attention nowadays.

Of course, as usual, a consistent formal derivation of multi-temperature fluid-dynamics as an asymptotic limit in collision dominated regime of a kinetic theory description would be highly desirable [5]. The task becomes quite heavy if one wants to include into the picture an essential ingredient for the above physical scenarios, like the occurrence of chemical reactions. Preliminary results in this direction were obtained in [2], but under very strong simplifying assumptions, mainly consideration of only translational degrees of freedom (monoatomic molecules). We shall consider here non-reactive mixtures, but we shall allow for an internal polyatomic structure, and subsequent additional degrees of freedom. In this way, there is exchange of internal energy in a collision, but without exchange of mass. The internal molecular structure may be taken into account by a discrete or continuous energy variable, as proposed in [10] and [6], respectively. Following the former discrete approach, the hydrodynamic limit of the Boltzmann equations depends crucially on the fast processes driving the evolution, whose collision operators are labeled by an inverse small parameter (typically, a Knudsen number). Some hydrodynamic regimes, even for the reactive case, were already considered in [1], but they were all leading to some single-temperature description. It is clear that, in order to avoid this restriction, one should be dealing with a gas in which equilibration within each single separate component runs faster than equalization of species parameters in the gas as a whole.

In [11] it was shown that, when the dominant operator is made up by all mechanical encounters preserving kinetic energy, including possible "resonant" collisions with change of internal state within the same species (as allowed by some form of degeneracy in the energy levels), hydrodynamic variables (apart from the usual mass densities) turn out to be given by a unique mass velocity and a unique translational temperature for the mixture, plus an internal (typically, vibrational) temperature for each species. The hydrodynamic limit is thus of multi-temperature type, though different from the previous ones. The slow gas-dynamic relaxation leads eventually to equalization of all temperatures, and, in case of reaction, to a mass action law for chemical equilibrium. A more detailed fluid-dynamic description could involve the presence of one mass velocity and of two temperatures for each species (one translational and one internal). We leave this formidable task to future research, and start addressing here the non-resonant problem, where vibrational temperatures disappear, but each species is endowed by its own velocity and (translational) temperature. In Sect. 2 it is assumed that fast collisions driving the overall evolution in our $Q$-component gas mixture are constituted by all mechanical encounters between particles of the same species. 
Conservation of mass, momentum, and energy is postulated in each scattering event of any type. Kinetic energy is thus not conserved in general, due to transitions of colliding particles from one energy state to another. The Euler equations for the $5 Q$ hydrodynamic variables (densities, velocities, and temperatures of each species) are derived and briefly discussed in Sect. 3. It is clear that this level of fluid-dynamic approximation represents a very simplified description of the physical problem under investigation, and has to be considered only as a first step in a complex asymptotic procedure that should lead eventually to more realistic hydrodynamic equations at Navier-Stokes level, featuring important dissipative effects like viscosity and heat flux. We hope to undertake this work in a near future. However, the present results constitute the preliminary but indispensable step for any further development, and their achievement requires, as we will see, significant amount of work in order to overcome nontrivial technical difficulties. In the present multi-temperature Euler equations, closed form analytical expressions for the slow collision contributions in the balance equations may be achieved under additional assumptions or in special situations (see Sect. 4), like Maxwell collision model, or only one energy level per species.

\section{Kinetic and Macroscopic Equations}

We shall start from the kinetic model for internal state transitions proposed in [10], in which each species $s, s=1, \ldots, Q$, is endowed with a structure of $N>1$ discrete energy levels, to mimic non-translational degrees of freedom. For a proper mathematical treatment, the $Q N$ different components are labeled according to a single index and ordered in such a way that the $s$-th chemical species may be regarded as the equivalence class of the indices $i$ which are congruent to $s$ modulo $Q$ (we shall write simply $i \equiv s$ ). If $A_{i}, 1 \leq i \leq Q N$, denotes the general component, and $E_{i}$ the corresponding energy of its state, the general binary interaction is written as $A_{i}+A_{j} \rightleftharpoons A_{h}+A_{k}$, and is described at Boltzmann level in terms of a cross section $\sigma_{i j}^{h k}$. We will denote by $\Delta E_{i j}^{h k}$ the net increase of internal energy $E_{h}+E_{k}-E_{i}-E_{j}$, whose gain or loss must be compensated by an opposite variation of the kinetic energies. Energies $E_{i} \geq 0$ are monotonically increasing with their index in the frame of each species, and all molecules $A_{i}$ with $i \equiv s$ share the same mass $m_{s}$. The kinetic equations for the evolution of the distribution functions $f_{i}(\mathbf{x}, \mathbf{v}, t)$ read [10]

$$
\begin{aligned}
\frac{\partial f_{i}}{\partial t}+\mathbf{v} \cdot \nabla_{\mathbf{x}} f_{i}=J_{i}[\underline{f]}= & \sum_{(j, h, k) \in D_{i}} \iint K_{i}^{i j h k}\left[\underline{f]}\left(\mathbf{v}, \mathbf{w}, \hat{\mathbf{n}}^{\prime}\right) d \mathbf{w} d \hat{\mathbf{n}}^{\prime}, \quad 1 \leq i \leq Q N\right. \\
K_{i}^{i j h k}[\underline{f}]\left(\mathbf{v}, \mathbf{w}, \hat{\mathbf{n}}^{\prime}\right)= & \Theta\left(g^{2}-\delta_{i j}^{h k}\right) B_{i j}^{h k}\left(g, \hat{\mathbf{n}} \cdot \hat{\mathbf{n}}^{\prime}\right) \\
& \times\left[\left(\frac{\mu_{i j}}{\mu_{h k}}\right)^{3} f_{h}\left(\mathbf{v}_{i j}^{h k}\right) f_{k}\left(\mathbf{w}_{i j}^{h k}\right)-f_{i}(\mathbf{v}) f_{j}(\mathbf{w})\right],
\end{aligned}
$$

where $g=|\mathbf{v}-\mathbf{w}|, \hat{\mathbf{n}}=(\mathbf{v}-\mathbf{w}) / g$, and $\mathbf{v}_{i j}^{h k}=\varepsilon_{i j} \mathbf{v}+\varepsilon_{j i} \mathbf{w}+\varepsilon_{k h} g_{i j}^{h k} \hat{\mathbf{n}}^{\prime}, \mathbf{w}_{i j}^{h k}=\varepsilon_{i j} \mathbf{v}+\varepsilon_{j i} \mathbf{w}-$ $\varepsilon_{h k} g_{i j}^{h k} \hat{\mathbf{n}}^{\prime}$, with $g_{i j}^{h k}=\left[\frac{\mu_{i j}}{\mu_{h k}}\left(g^{2}-\delta_{i j}^{h k}\right)\right]^{1 / 2}$. Here $\varepsilon_{i j}$ denotes the mass ratio $m_{i} /\left(m_{i}+m_{j}\right), \mu_{i j}=$ $\varepsilon_{i j} m_{j}$ the reduced mass, $B_{i j}^{h k}=g \sigma_{i j}^{h k}$ the collision kernel, while $\delta_{i j}^{h k}$ stands for $2 \Delta E_{i j}^{h k} / \mu_{i j}$; when the latter is positive, the unit step function $\Theta$ actually introduces a threshold for the collision. Moreover, the set $D_{i}$ is made up by all triplets $(j, h, k)$ with $h \equiv i$ and $k \equiv j$. The main properties of the collision operator $J_{i}$ (collision invariants, detailed balance, equilibria, $H$-theorem) are a byproduct of the detailed investigation performed in [10], where also all major moments, defined in the usual way, are listed. They include number density $n_{i}$ of 
each component and $N_{s}$ of each species, the corresponding drift velocities $\mathbf{u}_{i}$ and $\mathbf{u}_{s}$, total number density $n$, mass density $\rho$, mass velocity $\mathbf{u}$, pressure tensor $\mathbf{P}$, thermal energy density $U=\frac{1}{2} \operatorname{tr} \mathbf{P}$ (defining temperature $T$ as in a perfect gas), thermal heat flux $\mathbf{q}$, excitation energy density $U^{*}=\sum_{i=1}^{Q N} E_{i} n_{i}$, and excitation heat flux $\mathbf{q}^{*}$. We briefly recall here that there exist $Q+4$ independent collision invariants for the global collision operator, and they correspond to the species number densities $N_{s}$, the global mass velocity $\mathbf{u}$, and the total energy, sum of the kinetic contribution $\frac{1}{2} \rho u^{2}$ and of the internal one $U+U^{*}$. Consequently, $Q+4$ exact nonclosed macroscopic conservation equations hold

$$
\begin{aligned}
& \frac{\partial N_{s}}{\partial t}+\nabla_{\mathbf{x}} \cdot\left(N_{s} \mathbf{u}_{s}\right)=0 \quad s=1, \ldots, Q \\
& \frac{\partial}{\partial t}(\rho \mathbf{u})+\nabla_{\mathbf{x}} \cdot(\rho \mathbf{u} \otimes \mathbf{u}+\mathbf{P})=\mathbf{0} \\
& \frac{\partial}{\partial t}\left(\frac{1}{2} \rho u^{2}+U+U^{*}\right)+\nabla_{\mathbf{x}} \cdot\left[\left(\frac{1}{2} \rho u^{2}+U+U^{*}\right) \mathbf{u}+\mathbf{P} \cdot \mathbf{u}+\mathbf{q}+\mathbf{q}^{*}\right]=0,
\end{aligned}
$$

and collision equilibria are a $(Q+4)$-parameter family of Maxwellians with a common drift velocity and temperature

$$
\begin{aligned}
\mathcal{M}_{i}(\mathbf{v}) & =n_{i}\left(\frac{m_{s}}{2 \pi K T}\right)^{3 / 2} \exp \left[-\frac{m_{s}}{2 K T}(\mathbf{v}-\mathbf{u})^{2}\right] \quad \forall i \equiv s, \forall s=1, \ldots, Q \\
n_{i} & =\frac{N_{s}}{Z_{s}(T)} \exp \left(-\frac{E_{i}-E_{s}}{K T}\right), \quad Z_{s}(T)=\sum_{i \equiv s} \exp \left(-\frac{E_{i}-E_{s}}{K T}\right),
\end{aligned}
$$

where $K$ is the Boltzmann constant and $Z_{s}$ the partition function. A strict entropy inequality for relaxation to equilibrium can also be established in terms of the classical $H$-functional

$$
H=\sum_{s=1}^{Q} \sum_{i \equiv s} \int f_{i} \log f_{i} d \mathbf{v} .
$$

For the physical scenario described in the introduction, the typical mean free paths for collisions between components of the same species are much shorter than for collisions between components of different species, which in turn are of the same order as the macroscopic scale. Therefore the proper adimensionalization introduces an order parameter $\epsilon \ll 1$, representing the Knudsen number [5] for the "affine" collisions, the fast process, whose effects are dominant in the evolution problem. The scaled equations take then the form

$$
\frac{\partial f_{i}}{\partial t}+\mathbf{v} \cdot \nabla_{\mathbf{x}} f_{i}=\frac{1}{\epsilon} J_{i}^{F A}+J_{i}^{S L},
$$

where the superscripts $F A$ (fast) and $S L$ (slow) identify the sets, $D_{i}^{F A}$ and $D_{i}^{S L}$ respectively, to which the sums on the triplets $(j, h, k)$ in (1) must be restricted, namely

$$
\begin{aligned}
& D_{i}^{F A}=\left\{(j, h, k) \in D_{i}, i \equiv j \equiv h \equiv k\right\} \\
& D_{i}^{S L}=D_{i} \backslash D_{i}^{F A}=\left\{(j, h, k) \in D_{i}, i \not \equiv j, h \equiv i, k \equiv j\right\} .
\end{aligned}
$$

We are led thus to the analysis of the leading operators $J_{i}^{F A}$, which ignore the presence of interactions between different species. The study can be performed following standard procedures of kinetic theory (the interested reader may find some more details in [10] and [1]). 
Collision invariants are in number of $5 Q$, and may be chosen as

$$
\varphi_{i}(\mathbf{v})=a_{s}+\mathbf{b}_{s} \cdot m_{s} \mathbf{v}+c_{s}\left(\frac{1}{2} m_{s} v^{2}+E_{i}\right) \quad \forall i \equiv s, \forall s=1, \ldots, Q
$$

with arbitrary $a_{s}, c_{s} \in \mathbb{R}$ and $\mathbf{b}_{s} \in \mathbb{R}^{3}$. Since Boltzmann lemma and detailed balance principle are easily shown to hold, the "fast" collision equilibria are given by

$$
\begin{gathered}
f_{i}^{M}(\mathbf{v})=\frac{N_{s}}{Z_{s}\left(T_{s}\right)}\left(\frac{m_{s}}{2 \pi K T_{s}}\right)^{3 / 2} \exp \left[-\frac{m_{s}}{2 K T_{s}}\left(\mathbf{v}-\mathbf{u}_{s}\right)^{2}-\frac{E_{i}-E_{s}}{K T_{s}}\right] \\
\forall i \equiv s, \forall s=1, \ldots, Q
\end{gathered}
$$

each with a species density, velocity and temperature as free parameters, and with dependence on the state via $E_{i}$ and via the structure of the energy levels $E_{j}, j \equiv s$. Here $Z_{s}$ is the same as in (3), but computed at the species temperature $T_{s}$.

For any practical application one is mainly interested in the macroscopic equations which are obtained as weak forms of the kinetic equations when test functions are selected as the (independent) fast collision invariants. A convenient basis is provided by the three following options in (7), for each fixed $s=1, \ldots, Q: \mathbf{b}_{s}=\mathbf{0}, c_{s}=0 ; a_{s}=c_{s}=0 ; a_{s}=0, \mathbf{b}_{s}=\mathbf{0}$. They would represent conservation of mass, of momentum, and of (total) energy within each species. However, the resulting balance equations will not be of conservative type, due to the presence of the slow collision operator, which does not vanish under the above test functions (there is exchange of momentum and energy between different species). The general weak form reads as

$$
\begin{aligned}
\sum_{i=1}^{Q N} \int \varphi_{i}(\mathbf{v}) J_{i}^{S L} d \mathbf{v}= & \sum_{r \neq s} \sum_{i, h \equiv s} \sum_{j, k \equiv r} \iiint \varphi_{i}(\mathbf{v}) B_{i j}^{h k}\left(g, \hat{\mathbf{n}} \cdot \hat{\mathbf{n}}^{\prime}\right) \Theta\left(g^{2}-\delta_{i j}^{h k}\right) \\
& \times\left[f_{h}\left(\mathbf{v}_{i j}^{h k}\right) f_{k}\left(\mathbf{w}_{i j}^{h k}\right)-f_{i}(\mathbf{v}) f_{j}(\mathbf{w})\right] d \mathbf{v} d \mathbf{w} d \hat{\mathbf{n}}^{\prime}
\end{aligned}
$$

and, on using the Jacobian $d \mathbf{v}_{i j}^{h k} d \mathbf{w}_{i j}^{h k} d \hat{\mathbf{n}}=\left(g_{i j}^{h k} / g\right) d \mathbf{v} d \mathbf{w} d \hat{\mathbf{n}}^{\prime}$ and the microreversibility relation

$$
\left(1+\frac{\delta_{i j}^{h k}}{g^{2}}\right)^{1 / 2} B_{i j}^{h k}\left(g_{h k}^{i j}, \hat{\mathbf{n}} \cdot \hat{\mathbf{n}}^{\prime}\right)=B_{h k}^{i j}\left(g, \hat{\mathbf{n}} \cdot \hat{\mathbf{n}}^{\prime}\right),
$$

may be recast, after suitable rearrangements of sums and integrations, as

$$
\begin{aligned}
\sum_{i=1}^{Q N} \int \varphi_{i}(\mathbf{v}) J_{i}^{S L} d \mathbf{v}= & \sum_{r \neq s} \sum_{i, h \equiv s} \sum_{j, k \equiv r} \iiint\left[\varphi_{h}\left(\mathbf{v}_{i j}^{h k}\right)-\varphi_{i}(\mathbf{v})\right] \\
& \times B_{i j}^{h k}\left(g, \hat{\mathbf{n}} \cdot \hat{\mathbf{n}}^{\prime}\right) \Theta\left(g^{2}-\delta_{i j}^{h k}\right) f_{i}(\mathbf{v}) f_{j}(\mathbf{w}) d \mathbf{v} d \mathbf{w} d \hat{\mathbf{n}}^{\prime} .
\end{aligned}
$$

Now take for $\varphi_{i}$ the string which, once a species $s$ is assigned, shows 1 in all positions $i$ which are congruent to $s$, and zero elsewhere. Take the corresponding weak form of Eq. (5), and notice that, by virtue of Eq. (11), all collision contributions vanish (mass of each species is preserved also by slow collisions). Since $\rho_{s}=m_{s} N_{s}$ and $\rho_{s} \mathbf{u}_{s}=\sum_{i \equiv s} \rho_{i} \mathbf{u}_{i}$, we obtain simply the continuity equation for species $S$

$$
\frac{\partial N_{s}}{\partial t}+\nabla_{\mathbf{x}} \cdot\left(N_{s} \mathbf{u}_{s}\right)=0 \quad s=1, \ldots, Q
$$


This coincides of course with the first of (2). Notice that it is closed in the present framework.

Take then for $\varphi_{i}$ the string which, for given $s$, exhibits $m_{i} \mathbf{v}=m_{s} \mathbf{v}$ in all positions which are congruent to $s$, and zero otherwise. After a little algebra, the corresponding weak form of Eq. (5) takes the form

$$
\frac{\partial}{\partial t}\left(\rho_{s} \mathbf{u}_{s}\right)+\nabla_{\mathbf{x}} \cdot \sum_{i \equiv s}\left(\rho_{i} \mathbf{u}_{i} \otimes \mathbf{u}_{i}+\mathbf{P}_{i}\right)=\mathbf{R}_{s} \quad s=1, \ldots, Q
$$

with appearance of mass velocity and pressure tensor of each component of the considered species, and with a momentum exchange rate

$$
\mathbf{R}_{s}=\sum_{r \neq s} \sum_{i, h \equiv s} \sum_{j, k \equiv r} \iiint m_{s}\left(\mathbf{v}_{i j}^{h k}-\mathbf{v}\right) B_{i j}^{h k}\left(g, \hat{\mathbf{n}} \cdot \hat{\mathbf{n}}^{\prime}\right) \Theta\left(g^{2}-\delta_{i j}^{h k}\right) f_{i}(\mathbf{v}) f_{j}(\mathbf{w}) d \mathbf{v} d \mathbf{w} d \hat{\mathbf{n}}^{\prime}
$$

Finally, take for $\varphi_{i}$ the string which, for fixed $s$, exhibits $\frac{1}{2} m_{i} v^{2}+E_{i}$ in all positions which are congruent to $s$, and zero otherwise. Some longer manipulations lead to the energy balance equation for the considered species

$$
\begin{aligned}
& \frac{\partial}{\partial t}\left[\sum_{i \equiv s}\left(\frac{1}{2} \rho_{i} u_{i}^{2}+\frac{3}{2} n_{i} K T_{i}+E_{i} n_{i}\right)\right]+\nabla_{\mathbf{x}} \cdot\left\{\sum _ { i \equiv s } \left[\left(\frac{1}{2} \rho_{i} u_{i}^{2}+\frac{3}{2} n_{i} K T_{i}\right.\right.\right. \\
& \left.\left.\left.\quad+E_{i} n_{i}\right) \mathbf{u}_{i}+\mathbf{P}_{i} \cdot \mathbf{u}_{i}+\mathbf{q}_{i}\right]\right\}=S_{s} \quad s=1, \ldots, Q
\end{aligned}
$$

which involve additionally heat flux of each component of the species $s$, and with an energy exchange rate

$$
\begin{aligned}
S_{s}= & \sum_{r \neq s} \sum_{i, h \equiv s} \sum_{j, k \equiv r} \iiint\left\{\frac{1}{2} m_{s}\left[\left(v_{i j}^{h k}\right)^{2}-v^{2}\right]+E_{h}-E_{i}\right\} \\
& \times B_{i j}^{h k}\left(g, \hat{\mathbf{n}} \cdot \hat{\mathbf{n}}^{\prime}\right) \Theta\left(g^{2}-\delta_{i j}^{h k}\right) f_{i}(\mathbf{v}) f_{j}(\mathbf{w}) d \mathbf{v} d \mathbf{w} d \hat{\mathbf{n}}^{\prime} .
\end{aligned}
$$

The $5 Q$ exact, kinetic theory based, macroscopic balance equations are then expressed by (12), (13), (15), and are nonclosed with respect to the $5 Q$ hydrodynamic fields $N_{s}, \mathbf{u}_{s}, T_{s}$. Constitutive equations are needed for all fields which are relevant to single components of a species, in particular $\mathbf{P}_{i}$ and $\mathbf{q}_{i}$, and for the collision contributions on the right sides, (14) and (16), that involve the specific distribution functions $f_{i}$, and bear trace of their kinetic origin via the collision kernels affecting all integrals.

It can be noticed that, if all $\mathbf{R}_{s}$ are summed over $s$, we are allowed an additional substitution in the integrations and summations to be performed, namely $\mathbf{v} \leftrightarrow \mathbf{w}, \hat{\mathbf{n}} \leftrightarrow-\hat{\mathbf{n}}^{\prime}, i \leftrightarrow j$, $s \leftrightarrow r$, and this leads to a symmetrized form

$$
\begin{aligned}
\sum_{s=1}^{Q} \mathbf{R}_{s}= & \frac{1}{2} \sum_{s=1}^{Q} \sum_{r \neq s} \sum_{i, h \equiv s} \sum_{j, k \equiv r} \iiint\left(m_{s} \mathbf{v}_{i j}^{h k}+m_{r} \mathbf{w}_{i j}^{h k}-m_{s} \mathbf{v}-m_{r} \mathbf{w}\right) \\
& \times B_{i j}^{h k}\left(g, \hat{\mathbf{n}} \cdot \hat{\mathbf{n}}^{\prime}\right) \Theta\left(g^{2}-\delta_{i j}^{h k}\right) f_{i}(\mathbf{v}) f_{j}(\mathbf{w}) d \mathbf{v} d \mathbf{w} d \hat{\mathbf{n}}^{\prime} .
\end{aligned}
$$

An analogous symmetrized form may be obtained by summing up all $S_{s}$,

$$
\sum_{s=1}^{Q} S_{s}=\frac{1}{2} \sum_{s=1}^{Q} \sum_{r \neq s} \sum_{i, h \equiv s} \sum_{j, k \equiv r} \iiint\left[\frac{1}{2} m_{s}\left(v_{i j}^{h k}\right)^{2}+\frac{1}{2} m_{r}\left(w_{i j}^{h k}\right)^{2}-\frac{1}{2} m_{s} v^{2}\right.
$$




$$
\left.-\frac{1}{2} m_{r} w^{2}+\Delta E_{i j}^{h k}\right] B_{i j}^{h k}\left(g, \hat{\mathbf{n}} \cdot \hat{\mathbf{n}}^{\prime}\right) \Theta\left(g^{2}-\delta_{i j}^{h k}\right) f_{i}(\mathbf{v}) f_{j}(\mathbf{w}) d \mathbf{v} d \mathbf{w} d \hat{\mathbf{n}}^{\prime},
$$

so that microscopic conservations in the single collisions imply the expected overall macroscopic conservations of momentum and energy

$$
\sum_{s=1}^{Q} \mathbf{R}_{s}=\mathbf{0}, \quad \sum_{s=1}^{Q} S_{s}=0
$$

Consequently, summing over $s$ equations (13) and (15) yields consistently momentum and energy conservation for the gas mixture as expressed by the second and third conservation laws in (2).

\section{Hydrodynamic Euler Equations}

The lowest order hydrodynamic closure we are interested in here is now achieved by substituting the fast collision equilibria (8) for the distribution functions in all moments and integrals appearing in Eqs. (13) to (16). One gets rather easily for the moments

$$
\begin{aligned}
\sum_{i \equiv s} \rho_{i} \mathbf{u}_{i} \otimes \mathbf{u}_{i} & =\rho_{s} \mathbf{u}_{s} \otimes \mathbf{u}_{s}, \quad \sum_{i \equiv s} \mathbf{P}_{i}=N_{s} K T_{s} \mathbf{I}, \quad \sum_{i \equiv s} \mathbf{P}_{i} \cdot \mathbf{u}_{i}=N_{s} K T_{s} \mathbf{u}_{s}, \quad \mathbf{q}_{i}=\mathbf{0} \\
\sum_{i \equiv s} E_{i} n_{i} & =N_{s} \bar{E}_{s}\left(T_{s}\right), \quad \sum_{i \equiv s} E_{i} n_{i} \mathbf{u}_{i}=N_{s} \bar{E}_{s}\left(T_{s}\right) \mathbf{u}_{s},
\end{aligned}
$$

where the known function $\bar{E}_{s}\left(T_{s}\right)$ represents an equilibrium average of the energy states of the general species $s$

$$
\bar{E}_{s}\left(T_{s}\right)=\frac{1}{Z_{s}\left(T_{s}\right)} \sum_{i \equiv s} E_{i} \exp \left(-\frac{E_{i}-E_{s}}{K T_{s}}\right)
$$

and accounts for the correction to the energy law for the $s$-th molecules due to the nontranslational degrees of freedom.

Euler equations for the present model of a polyatomic gas mixture, for physical situations in which collisions between equal molecules are much more likely than any other event, read then as $(s=1, \ldots, Q)$

$$
\begin{aligned}
& \frac{\partial N_{s}}{\partial t}+\nabla_{\mathbf{x}} \cdot\left(N_{s} \mathbf{u}_{s}\right)=0 \\
& \frac{\partial}{\partial t}\left(\rho_{s} \mathbf{u}_{s}\right)+\nabla_{\mathbf{x}} \cdot\left(\rho_{s} \mathbf{u}_{s} \otimes \mathbf{u}_{s}\right)+\nabla_{\mathbf{x}}\left(N_{s} K T_{s}\right)=\hat{\mathbf{R}}_{s} \\
& \frac{\partial}{\partial t}\left(\frac{1}{2} \rho_{s} u_{s}^{2}+\frac{3}{2} N_{s} K T_{s}+N_{s} \bar{E}_{s}\left(T_{s}\right)\right) \\
& \quad+\nabla_{\mathbf{x}} \cdot\left[\left(\frac{1}{2} \rho_{s} u_{s}^{2}+\frac{5}{2} N_{s} K T_{s}+N_{s} \bar{E}_{s}\left(T_{s}\right)\right) \mathbf{u}_{s}\right]=\hat{S}_{s},
\end{aligned}
$$

where the exchange rates $\hat{\mathbf{R}}_{s}$ and $\hat{S}_{s}$ have become known functions of the $5 Q$ unknown fields (species densities, velocities and temperatures). 
A deep and detailed comparison of these results, obtained as asymptotic limit of a kinetic approach, with other models, especially from thermodynamics, is out of the goals of this paper, and will be matter of future work. However, we shall try to push analytical calculation of the production terms as far as possible in order to extract their main features and to analyze, at least in some simple cases, their trends versus the hydrodynamic fields.

To this end, we start writing $\hat{\mathbf{R}}_{s}=\sum_{r \neq s} \hat{\mathbf{R}}_{s r}$, with

$$
\begin{aligned}
\hat{\mathbf{R}}_{s r}= & -\mu_{s r} \sum_{i, h \equiv s} \sum_{j, k \equiv r} \iiint\left(\mathbf{g}-\sqrt{g^{2}-\delta_{i j}^{h k}} \hat{\mathbf{n}}^{\prime}\right) \\
& \times \Theta\left(g^{2}-\delta_{i j}^{h k}\right) B_{i j}^{h k}\left(g, \hat{\mathbf{n}} \cdot \hat{\mathbf{n}}^{\prime}\right) f_{i}^{M}(\mathbf{v}) f_{j}^{M}(\mathbf{w}) d \mathbf{v} d \mathbf{w} d \hat{\mathbf{n}}^{\prime},
\end{aligned}
$$

where integrations with respect to the unit vector $\hat{\mathbf{n}}^{\prime} \in S^{2}$ may be performed separately, leading to the angular moments of the collision kernel

$$
B_{i j}^{h k(\ell)}(g)=\int_{S^{2}}\left(\hat{\mathbf{n}} \cdot \hat{\mathbf{n}}^{\prime}\right)^{\ell} B_{i j}^{h k}\left(g, \hat{\mathbf{n}} \cdot \hat{\mathbf{n}}^{\prime}\right) d \hat{\mathbf{n}}^{\prime} \quad \ell=0,1 .
$$

Then, the product of the two Maxwellians can be cast as

$$
\begin{gathered}
f_{i}^{M}(\mathbf{v}) f_{j}^{M}(\mathbf{w})=\frac{N_{s}}{Z_{s}\left(T_{s}\right)} \frac{N_{r}}{Z_{r}\left(T_{r}\right)}\left(\frac{m_{s}}{2 \pi K T_{s}}\right)^{\frac{3}{2}}\left(\frac{m_{r}}{2 \pi K T_{r}}\right)^{\frac{3}{2}} \exp \left(-\frac{E_{i}-E_{s}}{K T_{s}}\right. \\
\left.-\frac{E_{j}-E_{r}}{K T_{r}}\right) \exp \left[-\alpha_{s r}\left(\mathbf{G}_{s r}+\gamma_{s r} \mathbf{g}-\boldsymbol{\delta}_{s r}\right)^{2}\right] \exp \left\{-\beta_{s r}\left[\mathbf{g}-\left(\mathbf{u}_{s}-\mathbf{u}_{r}\right)\right]^{2}\right\}
\end{gathered}
$$

where

$$
\begin{aligned}
& \alpha_{s r}=\frac{m_{s}}{2 K T_{s}}+\frac{m_{r}}{2 K T_{r}}, \quad \beta_{s r}=\left(\frac{2 K T_{s}}{m_{s}}+\frac{2 K T_{r}}{m_{r}}\right)^{-1} \\
& \gamma_{s r}=\frac{\mu_{s r}}{\alpha_{s r}}\left(\frac{1}{2 K T_{s}}-\frac{1}{2 K T_{r}}\right), \quad \boldsymbol{\delta}_{s r}=\frac{1}{\alpha_{s r}}\left(\frac{m_{s}}{2 K T_{s}} \mathbf{u}_{s}+\frac{m_{r}}{2 K T_{r}} \mathbf{u}_{r}\right)
\end{aligned}
$$

and $\mathbf{G}_{s r}$ is the center of mass velocity $\varepsilon_{s r} \mathbf{v}+\varepsilon_{r s} \mathbf{w}$. At this point, since $d \mathbf{v} d \mathbf{w}=d \mathbf{G}_{s r} d \mathbf{g}$, also the integration over $\mathbf{G}_{s r} \in \mathbb{R}^{3}$ may be performed explicitly, and one ends up with

$$
\begin{aligned}
\hat{\mathbf{R}}_{s r}= & -\mu_{s r} \frac{N_{s}}{Z_{s}\left(T_{s}\right)} \frac{N_{r}}{Z_{r}\left(T_{r}\right)}\left(\frac{\beta_{s r}}{\pi}\right)^{\frac{3}{2}} \sum_{i, h \equiv s} \sum_{j, k \equiv r} \exp \left(-\frac{E_{i}-E_{s}}{K T_{s}}-\frac{E_{j}-E_{r}}{K T_{r}}\right) \\
& \times \int_{\mathbb{R}^{3}} \Theta\left(g^{2}-\delta_{i j}^{h k}\right) \mathbf{g} \bar{B}_{i j}^{h k}(g) \exp \left\{-\beta_{s r}\left[\mathbf{g}-\left(\mathbf{u}_{s}-\mathbf{u}_{r}\right)\right]^{2}\right\} d \mathbf{g}
\end{aligned}
$$

where

$$
\bar{B}_{i j}^{h k}(g)=B_{i j}^{h k(0)}(g)-\left(1-\frac{\delta_{i j}^{h k}}{g^{2}}\right)^{\frac{1}{2}} B_{i j}^{h k(1)}(g) .
$$

Now it is easily realized that $\hat{\mathbf{R}}_{s r}=\mathbf{0}$ whenever $\mathbf{u}_{s}=\mathbf{u}_{r}$, and that it is a vector parallel to $\mathbf{u}_{s}-\mathbf{u}_{r}$ when $\mathbf{u}_{s} \neq \mathbf{u}_{r}$, i.e. $\hat{\mathbf{R}}_{s r}=-\mathfrak{X}_{s r} \hat{\mathbf{e}}_{s r}$, where $\hat{\mathbf{e}}_{s r}$ is the unit vector in the direction of 
$\mathbf{u}_{s}-\mathbf{u}_{r}$. This allows to push further analytical manipulations in polar coordinates, leaving only a one-dimensional integral with respect to $g=|\mathbf{g}|$

$$
\begin{aligned}
\mathfrak{X}_{s r}= & \frac{\mu_{s r}}{2 \sqrt{\pi}} \frac{1}{\beta_{s r}^{1 / 2}\left|\mathbf{u}_{s}-\mathbf{u}_{r}\right|^{2}} \frac{N_{s}}{Z_{s}\left(T_{s}\right)} \frac{N_{r}}{Z_{r}\left(T_{r}\right)} \\
& \times \sum_{i, h \equiv s} \sum_{j, k \equiv r} \exp \left(-\frac{E_{i}-E_{s}}{K T_{s}}-\frac{E_{j}-E_{r}}{K T_{r}}\right) \int_{0}^{\infty} \Theta\left(g^{2}-\delta_{i j}^{h k}\right) \bar{B}_{i j}^{h k}(g) \\
& \times\left\{\left(2 \beta_{s r}\left|\mathbf{u}_{s}-\mathbf{u}_{r}\right| g-1\right) \exp \left[-\beta_{s r}\left(g-\left|\mathbf{u}_{s}-\mathbf{u}_{r}\right|\right)^{2}\right]\right. \\
& \left.+\left(2 \beta_{s r}\left|\mathbf{u}_{s}-\mathbf{u}_{r}\right| g+1\right) \exp \left[-\beta_{s r}\left(g+\left|\mathbf{u}_{s}-\mathbf{u}_{r}\right|\right)^{2}\right]\right\} g d g .
\end{aligned}
$$

Of course the final integration depends on the specific form of the collision kernel $B_{i j}^{h k}$. However, setting $\Delta_{s r}=\beta_{s r}^{1 / 2}\left|\mathbf{u}_{s}-\mathbf{u}_{r}\right|$, we may write

$$
\begin{aligned}
\mathfrak{X}_{s r}= & \frac{\mu_{s r}}{2 \sqrt{\pi}} \frac{1}{\beta_{s r}^{3 / 2}\left|\mathbf{u}_{s}-\mathbf{u}_{r}\right|^{2}} \frac{N_{s}}{Z_{s}\left(T_{s}\right)} \frac{N_{r}}{Z_{r}\left(T_{r}\right)} \\
& \times \sum_{i, h \equiv s} \sum_{j, k \equiv r} \exp \left(-\frac{E_{i}-E_{s}}{K T_{s}}-\frac{E_{j}-E_{r}}{K T_{r}}\right) X_{i j}^{h k}\left(\left|\mathbf{u}_{s}-\mathbf{u}_{r}\right|, \beta_{s r}\right),
\end{aligned}
$$

where

$$
\begin{aligned}
X_{i j}^{h k}\left(\left|\mathbf{u}_{s}-\mathbf{u}_{r}\right|, \beta_{s r}\right)= & \int_{0}^{\infty} \Theta\left(x^{2}-\beta_{s r} \delta_{i j}^{h k}\right) \bar{B}_{i j}^{h k}\left(\beta_{s r}^{-1 / 2} x\right) \\
& \times\left\{\left(2 \Delta_{s r} x-1\right) \exp \left[-\left(x-\Delta_{s r}\right)^{2}\right]\right. \\
& \left.+\left(2 \Delta_{s r} x+1\right) \exp \left[-\left(x+\Delta_{s r}\right)^{2}\right]\right\} x d x
\end{aligned}
$$

We may conclude that $\hat{\mathbf{R}}_{s r}$ is a vector proportional to $\mathbf{u}_{s}-\mathbf{u}_{r}$, and that its component on that direction is linear in $N_{s}$ and $N_{r}$, and depends on the other hydrodynamic parameters only via $T_{s}, T_{r}$, and $\left|\mathbf{u}_{s}-\mathbf{u}_{r}\right|$. There is little hope to cast $X_{i j}^{h k}$ in closed analytical form, even for some simple model of the reduced collision kernel $\bar{B}_{i j}^{h k}$. Some special cases are discussed in the next section.

Similar steps are then in order for the energy exchange rate $\hat{S}_{s}=\sum_{r \neq s} \hat{S}_{s r}$, with

$$
\begin{aligned}
\hat{S}_{s r}= & -\mu_{s r} \sum_{i, h \equiv s} \sum_{j, k \equiv r} \iiint\left[\mathbf{G}_{s r} \cdot\left(\mathbf{g}-\sqrt{g^{2}-\delta_{i j}^{h k}} \hat{\mathbf{n}}^{\prime}\right)+\frac{1}{2} \varepsilon_{r s} \delta_{i j}^{h k}-\frac{E_{h}-E_{i}}{\mu_{s r}}\right] \\
& \times \Theta\left(g^{2}-\delta_{i j}^{h k}\right) B_{i j}^{h k}\left(g, \hat{\mathbf{n}} \cdot \hat{\mathbf{n}}^{\prime}\right) f_{i}^{M}(\mathbf{v}) f_{j}^{M}(\mathbf{w}) d \mathbf{v} d \mathbf{w} d \hat{\mathbf{n}}^{\prime} .
\end{aligned}
$$

After noticing that

$$
E_{h}-E_{i}-\frac{1}{2} \varepsilon_{r s} \mu_{s r} \delta_{i j}^{h k}=\varepsilon_{s r}\left(E_{h}-E_{i}\right)-\varepsilon_{r s}\left(E_{k}-E_{j}\right)
$$

integrations with respect to $\hat{\mathbf{n}}^{\prime}$ and $\mathbf{G}_{s r}$ may again be performed explicitly, and, skipping technical details, one ends up with an integral over $\mathbf{g}$, amenable to a one-dimensional integral over $g$ 


$$
\begin{aligned}
\hat{S}_{s r}= & \left(\frac{\beta_{s r}}{\pi}\right)^{3 / 2} \frac{N_{s}}{Z_{s}\left(T_{s}\right)} \frac{N_{r}}{Z_{r}\left(T_{r}\right)} \sum_{i, h \equiv s} \sum_{j, k \equiv r} \exp \left(-\frac{E_{i}-E_{s}}{K T_{s}}-\frac{E_{j}-E_{r}}{K T_{r}}\right) \\
& \times \int \Theta\left(g^{2}-\delta_{i j}^{h k}\right) \exp \left\{-\beta_{s r}\left[\mathbf{g}-\left(\mathbf{u}_{s}-\mathbf{u}_{r}\right)\right]^{2}\right\}\left\{\mu_{s r}\left(\gamma_{s r} g^{2}-\boldsymbol{\delta}_{s r} \cdot \mathbf{g}\right) \bar{B}_{i j}^{h k}(g)\right. \\
& \left.+\left[\varepsilon_{s r}\left(E_{h}-E_{i}\right)-\varepsilon_{r s}\left(E_{k}-E_{j}\right)\right] B_{i j}^{h k(0)}(g)\right\} d \mathbf{g} \\
= & \delta_{s r} \cdot \hat{\mathbf{R}}_{s r}+\left(\frac{\beta_{s r}}{\pi}\right)^{\frac{1}{2}} \frac{N_{s}}{Z_{s}\left(T_{s}\right)} \frac{N_{r}}{Z_{r}\left(T_{r}\right)} \sum_{i, h \equiv s} \sum_{j, k \equiv r} \exp \left(-\frac{E_{i}-E_{s}}{K T_{s}}-\frac{E_{j}-E_{r}}{K T_{r}}\right) \\
& \times \int \Theta\left(g^{2}-\delta_{i j}^{h k}\right)\left\{\mu_{s r} \gamma_{s r} g^{3} \bar{B}_{i j}^{h k}(g)+\left[\varepsilon_{s r}\left(E_{h}-E_{i}\right)-\varepsilon_{r s}\left(E_{k}-E_{j}\right)\right]\right. \\
& \left.\times g B_{i j}^{h k(0)}(g)\right\} \frac{\exp \left\{-\beta_{s r}\left[\mathbf{g}-\left(\mathbf{u}_{s}-\mathbf{u}_{r}\right)\right]^{2}\right\}-\exp \left\{-\beta_{s r}\left[\mathbf{g}+\left(\mathbf{u}_{s}-\mathbf{u}_{r}\right)\right]^{2}\right\}}{\left|\mathbf{u}_{s}-\mathbf{u}_{r}\right|} d g
\end{aligned}
$$

(for $\mathbf{u}_{s}=\mathbf{u}_{r}$ the fraction inside the integral must be replaced by its limiting value $\left.4 \beta_{s r} g \mathrm{e}^{-\beta_{s r} g^{2}}\right)$. The latter expression may be finally recast as

$$
\begin{aligned}
\hat{S}_{s r}= & \boldsymbol{\delta}_{s r} \cdot \hat{\mathbf{R}}_{s r}+\frac{1}{\sqrt{\pi}} \frac{1}{\Delta_{s r}} \frac{N_{s}}{Z_{s}\left(T_{s}\right)} \frac{N_{r}}{Z_{r}\left(T_{r}\right)} \\
& \times \sum_{i, h \equiv s} \sum_{j, k \equiv r} \exp \left(-\frac{E_{i}-E_{s}}{K T_{s}}-\frac{E_{j}-E_{r}}{K T_{r}}\right) Y_{i j}^{h k}\left(\left|\mathbf{u}_{s}-\mathbf{u}_{r}\right|, \beta_{s r}, \gamma_{s r}\right),
\end{aligned}
$$

where the integrals $Y_{i j}^{h k}$ depend also on a weighted temperature difference via $\gamma_{s r}$ :

$$
\begin{aligned}
Y_{i j}^{h k}= & \int_{0}^{\infty} \Theta\left(x^{2}-\beta_{s r} \delta_{i j}^{h k}\right)\left\{\mu_{s r} \gamma_{s r} \beta_{s r}^{-1} x^{3} \bar{B}_{i j}^{h k}\left(\beta_{s r}^{-1 / 2} x\right)\right. \\
& \left.+\left[\varepsilon_{s r}\left(E_{h}-E_{i}\right)-\varepsilon_{r s}\left(E_{k}-E_{j}\right)\right] x B_{i j}^{h k(0)}\left(\beta_{s r}^{-1 / 2} x\right)\right\} \\
& \times\left\{\exp \left[-\left(x-\Delta_{s r}\right)^{2}\right]-\exp \left[-\left(x+\Delta_{s r}\right)^{2}\right]\right\} d x
\end{aligned}
$$

Once more $\hat{S}_{s r}$ depends in general on the hydrodynamic variables $T_{s}$ and $T_{r}$ separately, and on velocities only through $\left|\mathbf{u}_{s}-\mathbf{u}_{r}\right|$.

\section{Exchange Rates for Simple Models}

If we assume isotropic Maxwell-molecule type of interaction for a given collision $(i, j) \rightarrow$ $(h, k)$, we may write

$$
\bar{B}_{i j}^{h k}(g)=B_{i j}^{h k(0)}(g)=\kappa_{i j}^{h k}=\mathrm{constant}
$$

and the integral $X_{i j}^{h k}$ in (31) reduces to

$$
\begin{aligned}
X_{i j}^{h k}= & \kappa_{i j}^{h k} \int_{\left(\beta_{s r} \delta_{i j}^{h k}\right)^{1 / 2}}^{\infty}\left\{\left(2 \Delta_{s r} x^{2}-x\right) \exp \left[-\left(x-\Delta_{s r}\right)^{2}\right]\right. \\
& \left.+\left(2 \Delta_{s r} x^{2}+x\right) \exp \left[-\left(x+\Delta_{s r}\right)^{2}\right]\right\} d x \quad \text { for } \delta_{i j}^{h k}>0
\end{aligned}
$$


or, by easy calculations, to

$$
X_{i j}^{h k}=2 \sqrt{\pi} \kappa_{i j}^{h k} \Delta_{s r}^{3} \quad \text { for } \delta_{i j}^{h k} \leq 0 .
$$

In the former case, patient manipulations lead to

$$
\begin{aligned}
X_{i j}^{h k}= & 2 \kappa_{i j}^{h k} \int_{\left(\beta_{s r} \delta_{i j}^{h k}\right)^{1 / 2}+\Delta_{s r}}^{\infty}\left(2 \Delta_{s r} z^{2}+2 \Delta_{s r}^{3}-\Delta_{s r}\right) \mathrm{e}^{-z^{2}} d z \\
& +\kappa_{i j}^{h k} \int_{\left(\beta_{s r} \delta_{i j}^{h k}\right)^{1 / 2}-\Delta_{s r}}^{\left(\beta_{s r} \delta_{i j}^{h k}\right)^{1 / 2}+\Delta_{s r}}\left[2 \Delta_{s r} z^{2}+\left(4 \Delta_{s r}^{2}-1\right) z+2 \Delta_{s r}^{3}-\Delta_{s r}\right] \mathrm{e}^{-z^{2}} d z
\end{aligned}
$$

so that everything is amenable to primitives of $\mathrm{e}^{-z^{2}}$ times the first few integer powers of $z$, namely to Gaussian and error functions, precisely

$$
\begin{aligned}
X_{i j}^{h k}= & 2 \sqrt{\pi} \kappa_{i j}^{h k}\left\{\Delta_{s r}^{3} \mathcal{F}_{1}\left(\beta_{s r}, \Delta_{s r}\right)-\left(\Delta_{s r}^{2}-\frac{1}{2}\right) \mathcal{F}_{2}\left(\beta_{s r}, \Delta_{s r}\right)\right. \\
& \left.+\Delta_{s r} \sqrt{\beta_{s r} \delta_{i j}^{h k}} \mathcal{F}_{3}\left(\beta_{s r}, \Delta_{s r}\right)\right\}
\end{aligned}
$$

for $\delta_{i j}^{h k}>0$, where

$$
\begin{aligned}
& \mathcal{F}_{1}\left(\beta_{s r}, \Delta_{s r}\right)=1-\frac{\operatorname{erf}\left(\sqrt{\beta_{s r} \delta_{i j}^{h k}}+\Delta_{s r}\right)+\operatorname{erf}\left(\sqrt{\beta_{s r} \delta_{i j}^{h k}}-\Delta_{s r}\right)}{2} \\
& \mathcal{F}_{2}\left(\beta_{s r}, \Delta_{s r}\right)=\frac{\mathrm{e}^{-\left(\sqrt{\beta_{s r} r_{i j}^{h k}}+\Delta_{s r}\right)^{2}}-\mathrm{e}^{-\left(\sqrt{\beta_{s r} \delta_{i j}^{h k}}-\Delta_{s r}\right)^{2}}}{2 \sqrt{\pi}} \\
& \mathcal{F}_{3}\left(\beta_{s r}, \Delta_{s r}\right)=\frac{\mathrm{e}^{-\left(\sqrt{\beta_{s r} \delta_{i j}^{h k}}+\Delta_{s r}\right)^{2}}+\mathrm{e}^{-\left(\sqrt{\beta_{s r} \delta_{i j}^{h k}}-\Delta_{s r}\right)^{2}}}{2 \sqrt{\pi}},
\end{aligned}
$$

recovering (38) for $\delta_{i j}^{h k} \rightarrow 0$. Expressions (38), (40), (42) make the dependence on $\beta_{s r}$ and on $\left|\mathbf{u}_{s}-\mathbf{u}_{r}\right|=\beta_{s r}^{-1 / 2} \Delta_{s r}$ explicit, if (36) holds. Unfortunately, this does not make explicit the whole exchange rate $\hat{\mathbf{R}}_{s}$. In fact, the sums in (30) involve, together with $X_{i j}^{h k}$, also its "reciprocal" $X_{h k}^{i j}$, for which the collision kernel is not Maxwellian anymore, because of the microreversibility condition (10). If we assume for instance that (36) holds for all $(i, j, h, k)$ such that $\Delta E_{i j}^{h k}>0$, then (40) is valid for all such $(i, j, h, k)$, but, since

$$
\bar{B}_{h k}^{i j}(g)=\kappa_{i j}^{h k}\left(1+\frac{\delta_{i j}^{h k}}{g^{2}}\right)^{1 / 2}
$$

we need to compute also, with $\delta_{i j}^{h k}>0$, the integrals

$$
\begin{aligned}
X_{h k}^{i j}= & \kappa_{i j}^{h k} \int_{0}^{\infty}\left(x^{2}+\beta_{s r} \delta_{i j}^{h k}\right)^{1 / 2}\left\{\left(2 \Delta_{s r} x-1\right) \exp \left[-\left(x-\Delta_{s r}\right)^{2}\right]\right. \\
& \left.+\left(2 \Delta_{s r} x+1\right) \exp \left[-\left(x+\Delta_{s r}\right)^{2}\right]\right\} d x
\end{aligned}
$$


which, with the presence of an irrational function of $x$, seems not to be amenable to any of the most common elementary or special functions.

A notable simplification occurs for monoatomic gases $(N=1)$, which is a kind of limiting situation, or toy model, for our physical system, but nevertheless can provide, as usual, some insight on the general problem, especially about dependence of the exchange rates on the hydrodynamic fields or combinations thereof. In particular, results can be useful for comparison with a general thermodynamic theory, based on first principles only, in whose frame they should not depend on the specific molecule, nor on the type of intermolecular potential. In the case of a single internal level, energy jumps are absent and sums are made up by a single term, so that momentum exchange rates collapse to

$$
\begin{aligned}
\hat{\mathbf{R}}_{s r}= & -\frac{1}{2 \sqrt{\pi}} \mu_{s r} N_{s} N_{r} \frac{\mathbf{u}_{s}-\mathbf{u}_{r}}{\Delta_{s r}^{3}} \int \bar{B}_{s r}^{s r}\left(\beta_{s r}^{-1 / 2} x\right) \\
& \times\left\{\left(2 \Delta_{s r} x-1\right) \exp \left[-\left(x-\Delta_{s r}\right)^{2}\right]\right. \\
& \left.+\left(2 \Delta_{s r} x+1\right) \exp \left[-\left(x+\Delta_{s r}\right)^{2}\right]\right\} x d x .
\end{aligned}
$$

For a Maxwellian collision model like (36), the final expression is very easy

$$
\hat{\mathbf{R}}_{s r}=-\mu_{s r} N_{s} N_{r} \kappa_{s r}^{s r}\left(\mathbf{u}_{s}-\mathbf{u}_{r}\right),
$$

but other collision models may allow explicit integration. The most significant one is probably the rigid sphere model

$$
\bar{B}_{s r}^{s r}(g)=\eta_{s r}^{s r} g
$$

and we quote here for illustrative purposes the final pair exchange rate (for $\mathbf{u}_{s} \neq \mathbf{u}_{r}$ )

$$
\begin{aligned}
\hat{\mathbf{R}}_{s r}= & -\frac{\eta_{s r}^{s r} \mu_{s r} N_{s} N_{r}}{\sqrt{\pi} \beta_{s r}^{1 / 2}}\left(\mathbf{u}_{s}-\mathbf{u}_{r}\right)\left[\left(\Delta_{s r}+\frac{1}{\Delta_{s r}}-\frac{1}{4 \Delta_{s r}^{3}}\right) \sqrt{\pi} \operatorname{erf}\left(\Delta_{s r}\right)\right. \\
& \left.+\left(1+\frac{1}{2 \Delta_{s r}^{2}}\right) \exp \left(-\Delta_{s r}^{2}\right)\right]
\end{aligned}
$$

in agreement with a result of [2]. The projection $\mathfrak{X}_{s r}$ depends here on velocities $\mathbf{u}_{s}$ and $\mathbf{u}_{r}$ only via $\left|\mathbf{u}_{s}-\mathbf{u}_{r}\right|$, and on temperatures $T_{s}$ and $T_{r}$ only via the combination $\frac{T_{s}}{m_{s}}+\frac{T_{r}}{m_{r}}$, whereas in (45) it was linear in $\left|\mathbf{u}_{s}-\mathbf{u}_{r}\right|$ and independent of temperatures. Limiting trends in (47) are

$$
\begin{aligned}
& \mathfrak{X}_{s r}=\frac{8}{3 \sqrt{\pi}} \frac{\eta_{s r}^{s r} \mu_{s r} N_{s} N_{r}\left|\mathbf{u}_{s}-\mathbf{u}_{r}\right|}{\beta_{s r}^{1 / 2}}+O\left(\left|\mathbf{u}_{s}-\mathbf{u}_{r}\right|^{3}\right) \quad \text { for }\left|\mathbf{u}_{s}-\mathbf{u}_{r}\right| \rightarrow 0 \\
& \mathfrak{X}_{s r} \sim \eta_{s r}^{s r} \mu_{s r} N_{s} N_{r}\left|\mathbf{u}_{s}-\mathbf{u}_{r}\right|^{2} \quad \text { for }\left|\mathbf{u}_{s}-\mathbf{u}_{r}\right| \rightarrow \infty
\end{aligned}
$$

Analogously, for the energy exchange rates, assuming isotropic Maxwellian molecules for a given $(i, j) \rightarrow(h, k)$ collision, as expressed by (36), the integral $Y_{i j}^{h k}$ in (35) becomes

$$
\begin{aligned}
Y_{i j}^{h k}= & \kappa_{i j}^{h k} \int_{\left(\beta_{s r} \delta_{i j}^{h k}\right)^{1 / 2}}^{\infty}\left\{\mu_{s r} \gamma_{s r} \beta_{s r}^{-1} x^{3}+\left[\varepsilon_{s r}\left(E_{h}-E_{i}\right)-\varepsilon_{r s}\left(E_{k}-E_{j}\right)\right] x\right\} \\
& \times\left\{\exp \left[-\left(x-\Delta_{s r}\right)^{2}\right]-\exp \left[-\left(x+\Delta_{s r}\right)^{2}\right]\right\} d x
\end{aligned}
$$


for $\delta_{i j}^{h k}>0$, while the lower integration bound must be replaced by 0 in the case $\delta_{i j}^{h k} \leq 0$. In the latter case manipulations are standard, though a bit lengthly, requiring essentially primitives of Gaussians times integer powers of $x$, and yield

$$
\begin{aligned}
Y_{i j}^{h k}= & \kappa_{i j}^{h k} \sqrt{\pi} \Delta_{s r}\left\{\mu_{s r}\left[\gamma_{s r}\left|\mathbf{u}_{s}-\mathbf{u}_{r}\right|^{2}-\frac{3 K\left(T_{s}-T_{r}\right)}{m_{s}+m_{r}}\right]\right. \\
& \left.+\varepsilon_{s r}\left(E_{h}-E_{i}\right)-\varepsilon_{r s}\left(E_{k}-E_{j}\right)\right\} \quad \text { for } \delta_{i j}^{h k} \leq 0
\end{aligned}
$$

with clear dependence on $T_{s}, T_{r}$ (also inside $\gamma_{s r}$ ) and on $\left|\mathbf{u}_{s}-\mathbf{u}_{r}\right|$, and with appearance of the actual temperature difference $T_{s}-T_{r}$.

Much more tedious and cumbersome is the computation for $\delta_{i j}^{h k}>0$, and we report here only the final result (recovering (50) if $\delta_{i j}^{h k} \rightarrow 0$ )

$$
\begin{aligned}
Y_{i j}^{h k}= & \kappa_{i j}^{h k} \mu_{s r} \gamma_{s r} \beta_{s r}^{-1} \sqrt{\pi}\left[\Delta_{s r}\left(\Delta_{s r}^{2}+\frac{3}{2}\right) \mathcal{F}_{1}\left(\beta_{s r}, \Delta_{s r}\right)\right. \\
& \left.+\left(1+\beta_{s r} \delta_{i j}^{h k}+\Delta_{s r}^{2}\right) \mathcal{F}_{2}\left(\beta_{s r}, \Delta_{s r}\right)+\sqrt{\beta_{s r} \delta_{i j}^{h k}} \Delta_{s r} \mathcal{F}_{3}\left(\beta_{s r}, \Delta_{s r}\right)\right] \\
& +\kappa_{i j}^{h k}\left[\varepsilon_{s r}\left(E_{h}-E_{i}\right)-\varepsilon_{r s}\left(E_{k}-E_{j}\right)\right] \sqrt{\pi}\left[\Delta_{s r} \mathcal{F}_{1}\left(\beta_{s r}, \Delta_{s r}\right)\right. \\
& \left.+\mathcal{F}_{2}\left(\beta_{s r}, \Delta_{s r}\right)\right] .
\end{aligned}
$$

For the same reasons as for $\hat{\mathbf{R}}_{s r}$, the actual computation of $\hat{S}_{s r}$ in closed form remains an open problem in general, even introducing as many Maxwell-molecules assumptions (36) as possible, since microreversibility renders the "reciprocal" collisions not manageable.

Once more, a considerable simplification occurs for monoatomic gases, in which case we have from (34)-(35)

$$
\begin{aligned}
\hat{S}_{s r}= & \delta_{s r} \cdot \hat{\mathbf{R}}_{s r}-\frac{2 \mu_{s r} N_{s} N_{r}}{\Delta_{s r} \sqrt{\pi}} \frac{K\left(T_{s}-T_{r}\right)}{m_{s}+m_{r}} \int_{0}^{\infty} x^{3} \bar{B}_{s r}^{s r}\left(\beta_{s r}^{-1 / 2} x\right) \\
& \times\left\{\exp \left[-\left(x-\Delta_{s r}\right)^{2}\right]-\exp \left[-\left(x+\Delta_{s r}\right)^{2}\right]\right\} d x .
\end{aligned}
$$

Now there are no jumps of internal energy and an overall assumption of Maxwell-type collision like (36) is consistent with microreversibility, yielding, after some simple algebra

$$
\begin{aligned}
\hat{S}_{s r} & =\delta_{s r} \cdot \hat{\mathbf{R}}_{s r}-\mu_{s r} N_{s} N_{r} \kappa_{s r}^{s r}\left(\frac{3 K\left(T_{s}-T_{r}\right)}{m_{s}+m_{r}}-\gamma_{s r}\left|\mathbf{u}_{s}-\mathbf{u}_{r}\right|^{2}\right) \\
& =-\mu_{s r} N_{s} N_{r} \kappa_{s r}^{s r}\left[\left(\varepsilon_{s r} \mathbf{u}_{s}+\varepsilon_{r s} \mathbf{u}_{r}\right) \cdot\left(\mathbf{u}_{s}-\mathbf{u}_{r}\right)+\frac{3 K\left(T_{s}-T_{r}\right)}{m_{s}+m_{r}}\right]
\end{aligned}
$$

which recovers a result from [2]. In this simplified case also other collision models can be worked out explicitly. For instance, for hard sphere interactions, we may use (46) and get

$$
\begin{aligned}
\hat{S}_{s r}= & -\mu_{s r} N_{s} N_{r} \eta_{s r}^{s r} \beta_{s r}^{-1 / 2} \\
& \times\left\{\frac{1}{\alpha_{s r}}\left[\left(\Delta_{s r}^{2}+1-\frac{1}{4 \Delta_{s r}^{2}}\right) \frac{\operatorname{erf}\left(\Delta_{s r}\right)}{\Delta_{s r}}+\left(1+\frac{1}{2 \Delta_{s r}^{2}}\right) \frac{\mathrm{e}^{-\Delta_{s r}^{2}}}{\sqrt{\pi}}\right]\right.
\end{aligned}
$$




$$
\begin{aligned}
& \times\left(\frac{m_{s}}{2 K T_{s}} \mathbf{u}_{s}+\frac{m_{r}}{2 K T_{r}} \mathbf{u}_{r}\right) \cdot\left(\mathbf{u}_{s}-\mathbf{u}_{r}\right)+\frac{2 K\left(T_{s}-T_{r}\right)}{m_{s}+m_{r}} \\
& \left.\times\left[\left(\Delta_{s r}^{4}+3 \Delta_{s r}^{2}+\frac{3}{4}\right) \frac{\operatorname{erf}\left(\Delta_{s r}\right)}{\Delta_{s r}}+\left(\Delta_{s r}^{2}+\frac{5}{2}\right) \frac{\mathrm{e}^{-\Delta_{s r}^{2}}}{\sqrt{\pi}}\right]\right\}
\end{aligned}
$$

with limiting trends

$$
\begin{aligned}
\hat{S}_{s r}= & -\mu_{s r} N_{s} N_{r} \eta_{s r}^{s r} \beta_{s r}^{-1 / 2} \frac{8}{3 \sqrt{\pi}}\left[\frac{3 K\left(T_{s}-T_{r}\right)}{m_{s}+m_{r}}+\frac{1}{\alpha_{s r}}\left(\frac{m_{s}}{2 K T_{s}} \mathbf{u}_{s}+\frac{m_{r}}{2 K T_{r}} \mathbf{u}_{r}\right)\right. \\
& \left.\cdot\left(\mathbf{u}_{s}-\mathbf{u}_{r}\right)+O\left(\left|\mathbf{u}_{s}-\mathbf{u}_{r}\right|^{2}\right)\right] \text { for }\left|\mathbf{u}_{s}-\mathbf{u}_{r}\right| \rightarrow 0 \\
\hat{S}_{s r} \sim & -\mu_{s r} N_{s} N_{r} \eta_{s r}^{s r} \beta_{s r} \frac{2 K\left(T_{s}-T_{r}\right)}{m_{s}+m_{r}}\left|\mathbf{u}_{s}-\mathbf{u}_{r}\right|^{3} \quad \text { for }\left|\mathbf{u}_{s}-\mathbf{u}_{r}\right| \rightarrow \infty .
\end{aligned}
$$

Above results about the dependence of the exchange rates on the hydrodynamic variables seem, at least qualitatively, not to be directly amenable to trends predicted, in a different frame, starting from basic principles of thermodynamics [19]. Quantifying and understanding the relevant discrepancies is scheduled as future investigation.

Acknowledgements Work performed in the frame of activities sponsored by INdAM-GNFM and by the Universities of Parma and Milan. Helpful discussions with L. Desvillettes and S. Simic are also gratefully acknowledged.

\section{References}

1. Bisi, M., Groppi, M., Spiga, G.: Kinetic modelling of bimolecular chemical reactions. In: Toscani, G. (ed.) Kinetic Methods for Nonconservative and Reacting Systems. Quaderni di Matematica, vol. 16, pp. 1-143. Aracne Editrice, Roma (2005)

2. Bisi, M., Martaló, G., Spiga, G.: Multi-temperature Euler hydrodynamics for a reacting gas from a kinetic approach to rarefied mixtures with resonant collisions. Europhys. Lett. 95, 55002 (2011)

3. Bose, T.K.: High Temperature Gas Dynamics. Springer, Berlin (2003)

4. Brini, F.: On the flame structure in multi-temperature mixture theory. Ric. Mat. 58, 63-76 (2009)

5. Cercignani, C.: Rarefied Gas Dynamics. From Basic Concepts to Actual Calculations. Cambridge University Press, Cambridge (2000)

6. Desvillettes, L., Monaco, R., Salvarani, F.: A kinetic model allowing to obtain the energy law of polytropic gases in the presence of chemical reactions. Eur. J. Mech. B, Fluids 24, 219-236 (2005)

7. Goebel, C.J., Harris, S.M., Johnson, E.A.: Two-temperature disparate-mass gas mixtures: a thirteen moment description. Phys. Fluids 19, 627-635 (1976)

8. Goldman, E., Sirovich, L.: Equations for mixtures. Phys. Fluids 10, 1928-1940 (1967)

9. Gouin, H., Ruggeri, T.: Identification of an average temperature and a dynamical pressure in a multitemperature mixture of fluids. Phys. Rev. E 78, 016303 (2008)

10. Groppi, M., Spiga, G.: Kinetic approach to chemical reactions and inelastic transitions in a rarefied gas. J. Math. Chem. 26, 197-219 (1999)

11. Groppi, M., Spiga, G., Zus, F.: Euler closure of the Boltzmann equations for resonant bimolecular reactions. Phys. Fluids 18, 057105 (2006)

12. Kustova, E.V., Nagnibeda, E.A.: On a correct description of a multi-temperature dissociating $\mathrm{CO}_{2}$ flow. Chem. Phys. 321, 293-310 (2006)

13. Matsuk, V.A., Rykov, V.A.: The Chapman-Enskog method for a multivelocity multitemperature reacting gas mixture. U.S.S.R. Comput. Math. Math. Phys. 18, 154-166 (1978)

14. Müller, I., Ruggeri, T.: Rational Extended Thermodynamics. Springer, New York (1998)

15. Park, C.: Nonequilibrium Hypersonic Aerothermodynamics. Wiley, New York (1990)

16. Ramshaw, J.D.: Hydrodynamic theory of multicomponent diffusion and thermal diffusion in multitemperature gas mixtures. J. Non-Equilib. Thermodyn. 18, 121-134 (1993) 
17. Ruggeri, T.: Multi-temperature mixture of fluids. Theor. Appl. Mech. 36, 207-238 (2009)

18. Ruggeri, T., Lou, J.: Heat conduction in multi-temperature mixtures of fluids: the role of the average temperature. Phys. Lett. A 373, 3052-3055 (2009)

19. Ruggeri, T., Simic, S.: On the hyperbolic system of a mixture of Eulerian fluids: a comparison between single- and multi-temperature models. Math. Methods Appl. Sci. 30, 827-849 (2007)

20. Ruggeri, T., Simic, S.: Average temperature and Maxwellian iteration in multitemperature mixtures of fluids. Phys. Rev. E 80, 026317 (2009)

21. Struminskii, V.V., Shavaliyev, M.Sh.: Transport phenomena in multivelocity, multitemperature gas mixtures. J. Appl. Math. Mech. 50, 59-64 (1986)

22. Weinert, U., Suchy, K.: Generalization of the moment method of Maxwell-Grad for multi-temperature gas mixtures and plasmas. Z. Naturfosch. 32a, 390-400 (1977) 